\title{
Cannabinoids in Glioblastoma Therapy: New Applications for Old Drugs
}

\author{
Claudia A. Dumitru ${ }^{1}$, I. Erol Sandalcioglu ${ }^{1}$ and Meliha Karsak ${ }^{2 *}$ \\ ${ }^{1}$ Department of Neurosurgery, KRH Klinikum Nordstadt, Nordstadt Hospital Hannover, Hannover, Germany, ${ }^{2}$ Neuronal and \\ Cellular Signal Transduction, Center for Molecular Neurobiology Hamburg (ZMNH), University Medical Center \\ Hamburg-Eppendorf (UKE), Hamburg, Germany
}

OPEN ACCESS

Edited by:

Ildikó Rácz,

Universitätsklinikum Bonn, Germany

Reviewed by:

Ethan Budd Russo, PHYTECS, United States

Miriam Sciaccaluga,

University of Perugia, Italy

*Correspondence:

Meliha Karsak

meliha.karsak@zmnh.uni-

hamburg.de;

mkarsak@uke.de

Received: 25 February 2018 Accepted: 25 April 2018 Published: 16 May 2018

Citation: Dumitru CA, Sandalcioglu IE and Karsak M (2018) Cannabinoids in Glioblastoma Therapy: New Applications for Old Drugs.

Front. Mol. Neurosci. 11:159. doi: 10.3389/fnmol.2018.00159
Glioblastoma (GBM) is the most malignant brain tumor and one of the deadliest types of solid cancer overall. Despite aggressive therapeutic approaches consisting of maximum safe surgical resection and radio-chemotherapy, more than 95\% of GBM patients die within 5 years after diagnosis. Thus, there is still an urgent need to develop novel therapeutic strategies against this disease. Accumulating evidence indicates that cannabinoids have potent anti-tumor functions and might be used successfully in the treatment of GBM. This review article summarizes the latest findings on the molecular effects of cannabinoids on GBM, both in vitro and in (pre-) clinical studies in animal models and patients. The therapeutic effect of cannabinoids is based on reduction of tumor growth via inhibition of tumor proliferation and angiogenesis but also via induction of tumor cell death. Additionally, cannabinoids were shown to inhibit the invasiveness and the stem cell-like properties of GBM tumors. Recent phase II clinical trials indicated positive results regarding the survival of GBM patients upon cannabinoid treatment. Taken together these findings underline the importance of elucidating the full pharmacological effectiveness and the molecular mechanisms of the cannabinoid system in GBM pathophysiology.

\footnotetext{
Keywords: cannabinoids, glioblastoma, molecular mechanisms, novel therapeutic strategies, cannabinoid receptors, cannabidiol, THC
}

\section{GLIOBLASTOMA}

Gliomas are the most common primary tumors of the central nervous system. Half of the newly-diagnosed gliomas are glioblastomas (GBMs), with an incidence in adults of 0.59-3.69 cases per 100,000 person life-years (Ostrom et al., 2014). The vast majority of GBM develop de novo (primary GBM); however, GBM can also evolve from lower grade gliomas (secondary GBM). Primary GBM occur more commonly in male patients whereas the reverse is the case for secondary GBM (Adamson et al., 2009). The mean age of primary and secondary GBM patients is 62 and 45 years, respectively (Adamson et al., 2009).

GBM is an extremely aggressive type of cancer. These tumors are characterized by high cellular proliferation and angiogenesis resulting in rapid tumor growth and, consequently, necrosis. GBM cells also exhibit high migration and invasive properties, which allow them to produce metachronous lesions and even to spread through the brain parenchyma. Furthermore, GBM tumors contain a subpopulation of glioma stem-like cells (GSCs), which, at least partially, account for the high resistance to therapy and recurrence rates of these tumors (Louis et al., 2016). 
Currently, the standard of care treatment for GBM consists of maximum safe surgical resection followed by radiotherapy plus concomitant and adjuvant chemotherapy with temozolomide (TMZ; Stupp et al., 2005). Despite this aggressive therapeutic regimen, GBM patients have a poor prognosis, with only $0.05 \%-4.7 \%$ of patients surviving 5 years past initial diagnosis (Ostrom et al., 2014). Recent advances in molecular pathology identified various GBM subtypes and thus, paved the way for more individualized therapeutic strategies. However, GBM remains incurable at present and there is still an urgent need to further characterize and target the molecular mechanisms involved in its progression.

\section{CANNABINOIDS}

The term "cannabinoids" originally described bioactive constituents of the plant Cannabis sativa. The cannabis ingredients were used traditionally for their medicinal purpose but also for their recreational properties. In addition to the psychoactive cannabinoid $\Delta^{9}$-tetrahydrocannabinol (THC), a number of other phytocannabinoids have been successfully extracted such as cannabinol, cannabidiol (CBD), cannabigerol or the flavoring agent beta-caryophyllene (BCP; Mechoulam, 1970; Gertsch et al., 2008). Most of the cannabinoids bind to G-protein coupled cannabinoid receptors, $\mathrm{CB} 1$ and $\mathrm{CB} 2$, and act as agonists or inverse agonists. Of special interest for therapeutic purposes are cannabinoids that are absent of intoxicating effects such as the $\mathrm{CB} 2$-selective $\mathrm{BCP}$ and $\mathrm{CBD}$ (Sharma et al., 2016; Russo, 2017). The cannabis constituent CBD has no significant agonistic activity on cannabinoid receptors (Howlett et al., 2002; Pertwee, 2005) however it targets a number of G-protein coupled receptors like GPR12, GPR6, GPR3, GPR55 and 5-HT1A and also transient receptor potential vanilloid TRPV1 and TRPV2 (Espejo-Porras et al., 2013; Nabissi et al., 2013; Hassan et al., 2014; Brown et al., 2017; Kaplan et al., 2017; Laun and Song, 2017). Cannabinoid receptors can also be selectively activated by pharmacologically efficient synthetic cannabinoids. Furthermore, cannabinoid receptors are activated by endogenously-produced arachidonic acid derivatives. The so-called endocannabinoids, anandamide and 2-arachidonoylglycerol (2-AG), are synthesized from cell membrane phospholipids by specific enzymes. In GBM, increased levels of anandamide and reduced activity of the synthesizing enzyme $\mathrm{N}$-acylglycerol phosphatidylethanolamine-phospholipase D (NAPE-PLD) and degrading enzyme fatty acid amide hydrolase (FAAH) have been identified (Petersen et al., 2005).

The activation of G-alpha i/o-coupled cannabinoid receptors inhibits adenylate cyclases, signals via ceramide, and induces kinase phosphorylation of focal adhesion kinase (FAK), mitogenactivated protein kinase (MAPK), and phosphatidylinositol3-kinase (PI3K). Cannabinoid receptors also regulate the expression of immediate early genes and regulate the production of nitric oxide (Howlett et al., 2002). Additionally, certain voltage dependent calcium and inwardly rectifying potassium channels can be modulated via cannabinoid receptor signaling ( $\mathrm{Lu}$ and Mackie, 2016). Thus, activation of CB1 or CB2 receptors exerts diverse consequences on cellular biology and functions ( $\mathrm{Lu}$ and Mackie, 2016).

\section{MOLECULAR MECHANISMS OF CANNABINOIDS IN GBM}

GBM tumors are known to express both major cannabinoidspecific receptors $\mathrm{CB} 1$ and $\mathrm{CB} 2$. The expression of these receptors has been detected in GBM cell lines, in ex-vivo primary tumor cells derived from GBM patients and in situ, in GBM tissue biopsies. There is a general consensus that high-grade gliomas, including GBM, express high levels of CB2. Furthermore, CB2 expression positively correlates with the malignancy grade (reviewed in Ellert-Miklaszewska et al., 2013). In contrast, the expression of CB1 still requires characterization, as it has been reported to be either unchanged (Schley et al., 2009), decreased (De Jesús et al., 2010) or even increased (Wu et al., 2012; Ciaglia et al., 2015) in GBM compared to low-grade gliomas or non-tumor control tissues.

The identification of altered expression of cannabinoid receptors in gliomas and GBM led to the hypothesis that cannabinoid receptor agonists might be used as anticancer agents. Indeed, a pilot clinical study was already developed more than a decade ago to investigate the anti-tumor activity of THC in patients with glioma. The study held promising results as it showed a decrease of tumor cell proliferation upon administration of THC in two of nine patients (Guzmán et al., 2006). Since then, an increasing number of studies sought to elucidate the molecular mechanisms triggered via the cannabinoids-cannabinoid receptors axis in gliomas and GBM. The major findings are described below and a summary is provided in Figure 1.

\section{CANNABINOIDS AND GBM TUMOR GROWTH}

The best studied effect of cannabinoids on GBM pathophysiology is the inhibition of tumor growth. A number of in vivo studies demonstrated that cannabinoids could significantly reduce tumor volume in orthotopic and subcutaneous animal models of glioma (for a comprehensive review, see Rocha et al., 2014). The mechanisms mediating this phenomenon can be roughly grouped into three categories: (1) cell death-inducing mechanisms (apoptosis and cytotoxic autophagy); (2) cell proliferation-inhibiting mechanisms; and (3) anti-angiogenic mechanisms.

Cannabinoid-induced cell death occurs mainly through the intrinsic (mitochondria-dependent) apoptotic pathway (reviewed in Ellert-Miklaszewska et al., 2013). Briefly, the pro-apoptotic Bcl-2 family member Bad is phosphorylated in response to cannabinoid treatment and translocates to the mitochondria. This results in loss of integrity of the outer mitochondrial membrane, release of cytochrome $\mathrm{c}$ and activation of apoptosis-executioner caspases. The activation of the intrinsic apoptosis pathway by cannabinoids is thought to be mediated by an increase in intracellular ceramide which, in turn, inhibits the pro-survival pathways PI3K/Akt and Raf1/MEK/ERK thereby 


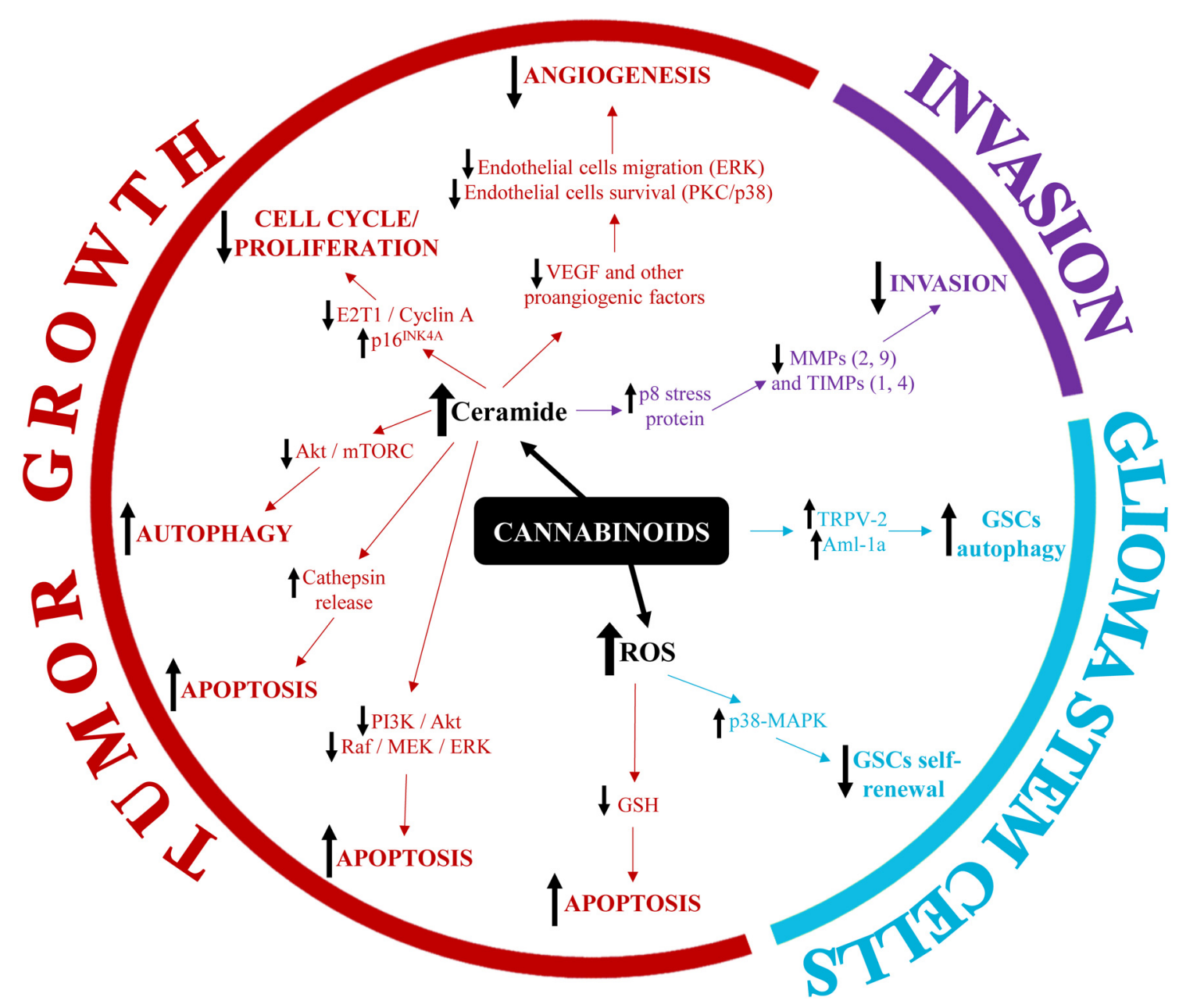

FIGURE 1 | Summary of the main molecular mechanisms involved in the modulation of glioblastoma (GBM) pathophysiology by cannabinoids.

allowing Bad to translocate to the mitochondria. Interestingly, ceramide has been also implicated in cannabinoid-induced autophagy of glioma cells through the p8/TRB3 pathway and subsequent inhibition of the Akt/mTORC1 axis (Carracedo et al., 2006; Salazar et al., 2009). Recent studies additionally showed that THC altered the balance between ceramides and dihydroceramides in autophagosomes and autolysosomes, which promoted the permeabilization of the organellar membrane, the release of cathepsins in the cytoplasm and the subsequent activation of apoptotic cell death (Hernández-Tiedra et al., 2016).

In addition to ceramide-mediated cell death, cannabinoids were also shown to trigger apoptosis via oxidative stress (reviewed in Massi et al., 2010). Specifically, glioma cells treated with CBD responded with reactive oxygen species (ROS) production, GSH depletion and caspase-9, -8 and -3 activation. Furthermore, combined treatment of GBM cells with THC and CBD induced a significant increase in the formation of ROS, which was linked to a later induction of apoptosis (Marcu et al., 2010). Recently however, Scott et al. (2015) showed that, while CBD treatment of glioma cells did induce a significant increase in ROS production, this phenomenon was accompanied by an upregulation of a large number of genes belonging to the heat-shock protein (HSP) super-family. As the subsequent upregulation of HSP client proteins diminished the cytotoxic effect of $\mathrm{CBD}$, the authors proposed that the inclusion of HSP inhibitors might enhance the anti-tumor effects of cannabinoids in glioma/GBM treatment regimens (Scott et al., 2015).

Apart from a direct killing effect on tumor cells, cannabinoids can also induce cell cycle arrest thereby inhibiting tumor cell proliferation. For instance, treatment of GBM cells with THC and/or CBD increases the population of cells in the $G_{0}-G_{1}$ phase and $G_{2}-G_{M}$ phase while decreasing the number of cells in the S-phase (Marcu et al., 2010). Similarly, Galanti et al. (2008) found that administration of THC to human GBM cell lines induced $\mathrm{G}_{0}-\mathrm{G}_{1}$ phase arrest. The authors also characterized some of the molecular mechanisms involved in cannabinoid-induced cell cycle arrest and found that THC decreased the levels of E2F1 and Cyclin A (two proteins that promote cell cycle progression) while upregulating the level of the cell cycle inhibitor p16 ${ }^{\text {INK4A }}$ (Galanti et al., 2008). 
The inhibitory effects of cannabinoids on GBM growth are, however, not restricted to the direct modulation of tumor cell death/survival or proliferation pathways. Several studies showed that cannabinoids were also able to inhibit tumor angiogenesis. For instance, Blázquez et al. (2003) found that local administration of the nonpsychotic cannabinoid JWH-133 to mice inhibited angiogenesis of malignant gliomas, since the cannabinoid-treated tumors had a small, differentiated and impermeable vasculature while the vasculature of the control tumors was large, plastic and leaky (Blázquez et al., 2003). The same group later demonstrated that local administration of THC resulted in a decrease of pro-angiogenic VEGF levels in two patients with recurrent GBM (Blázquez et al., 2004). In vitro, cannabinoids inhibited endothelial cell migration via the ERK pathway and endothelial cell survival via protein kinase C (PKC) and p38-MAPK pathways (Blázquez et al., 2003). Similarly, Solinas et al. (2012) demonstrated that CBD induced endothelial cell cytostasis, inhibited endothelial cell migration and sprouting in vitro and inhibited angiogenesis in vivo. These effects were accompanied by a downregulation of pro-angiogenic factors such as matrix metalloprotease- 2 and -9 (MMP2 and MMP9), urokinase-type plasminogen activator (uPA), endothelin-1 (ET-1), platelet-derived growth factor-AA (PDGF-AA) and chemokine (c-X-c motif) ligand 16 (CXCL16; Solinas et al., 2012).

While most studies found that the agonistic stimulation via $\mathrm{CB}$ receptors is responsible for the anti-tumor effects of cannabinoids, recent evidence suggests that $\mathrm{CB} 1$ antagonists might also be useful in glioma therapy. Specifically, Ciaglia et al. (2015) found that the pharmacological inactivation of CB1 by SR141716 inhibited glioma cell growth through cell cycle arrest and induction of caspase-dependent apoptosis. Interestingly however, SR141716 additionally upregulated the expression of NKG2D ligands (MICA and MICB) on the surface of glioma cells via STAT3 inactivation. The increase of MICA/B levels subsequently enhanced the recognition and killing of glioma cells by NK-cells. Notably, SR141716-induced MICA/B upregulation directly correlated with the degree of CB1 expression and occurred only in malignant glioma cells but not in normal human astrocytes (Ciaglia et al., 2015). Taken together these findings suggest that $\mathrm{CB} 1$ specific antagonists might be useful in multimodal therapeutic strategies, at least for certain subsets of GBM with high expression of CB1.

\section{CANNABINOIDS AND GBM INVASION}

Although gliomas and GBM rarely metastasize, these tumor cells are very adept at infiltrating the surrounding healthy brain tissue and spreading through the brain parenchyma (reviewed in Manini et al., 2018). Therefore, therapeutic strategies aimed at inhibiting the migration and invasion of GBM cells are of great clinical relevance in the management of this disease.

The role of cannabinoids in GBM migration and invasion is still poorly characterized. Nevertheless, accumulating evidence suggests that cannabinoids have potent anti-invasive effects on glioma cells both in vitro and in vivo. For instance, Soroceanu et al. (2013) showed that CBD inhibited the invasion of
GBM cells through organotypic brain slices. This anti-invasive effect was attributed to the inhibition of Id-1 expression by $\mathrm{CBD}$ and was observed in several GBM cell lines, in ex-vivo primary GBM cells and in an orthotopic xenograft murine model (Soroceanu et al., 2013). Solinas et al. found that CBD significantly inhibited GBM invasion even at low concentrations, which were otherwise not sufficient to induce tumor cell death (Solinas et al., 2013). The authors further demonstrated that CBD treatment of GBM cells significantly downregulated major proteins associated with tumor invasion, in particular MMP-9 and TIMP-4 (Solinas et al., 2013). Moreover additional MMPs and TIMPs have been linked to the anti-invasive effects of cannabinoids in glioma. Specifically, both TIMP-1 and MMP-2 were downregulated by THC treatment of glioma cells. These effects were mediated via ceramide accumulation and activation of $\mathrm{p} 8$ stress protein and, interestingly, were observed in glioma bearing mice as well as in two patients with recurrent GBM who had received intra-tumor injections with THC (Blázquez et al., 2008a,b).

\section{CANNABINOIDS AND GLIOMA STEM-LIKE CELLS (GSCs)}

A major challenge for GBM treatment is the resistance of the recurrent tumor to therapy. Accumulating evidence indicates that a subpopulation of GSCs contributes to this phenomenon through multiple mechanisms, such as alteration of DNA damage response, hypoxic microenvironment, Notch signaling pathway or multidrug resistance (reviewed in Liebelt et al., 2016).

GSCs express both major cannabinoid receptors, CB1 and CB2, as well as other components of the endocannabinoid system (Aguado et al., 2007). Exploratory gene array studies found that cannabinoid agonists altered the expression of genes involved in stem cell proliferation and differentiation. Cannabinoid-treated GSCs responded with increased S-100ß and GFAP expression and with simultaneous downregulation of the neuroepithelial progenitor marker nestin. Furthermore, cannabinoid challenge reduced the efficiency of GSCs to initiate glioma formation in vivo, as indicated by decreased neurosphere formation and cell proliferation in secondary xenografts (Aguado et al., 2007). The differentiation of GSCs was recently linked to the expression levels of the transcription factor Aml-1a. Nabissi et al. (2015) found that Aml-1a was upregulated during GSCs differentiation while Aml-1a knock-down restored a stem-cell phenotype in differentiated GSCs. Interestingly, treatment of GSCs with CBD upregulated the expression of Aml-1a in a TRPV2- and PI3K/Akt-dependent manner thereby inducing autophagy and abrogating the chemoresistance of GSCs to BCNU therapy (Nabissi et al., 2015).

Another potential mechanism regulating the "stemness" of GSCs upon cannabinoid treatment involves the intracellular increase of ROS. Specifically, CBD was shown to inhibit the self-renewal of GSCs via activation of the p38-MAPK pathway and downregulation of key stem cell mediators such as Sox2, Id1 and p-STAT3, while co-treatment with antioxidants abrogated these effects. In vivo, treatment of intracranial GSCs-derived tumors with CBD inhibited tumor 
cell proliferation, activated the pro-apoptotic caspase- 3 and significantly prolonged the survival of tumor-bearing mice. Even though a subset of GSCs adapted to CBD treatment and led to tumor regrowth, this phenomenon could be abrogated by combined therapy with CBD and small molecule modulators of ROS (Singer et al., 2015).

\section{CLINICAL RELEVANCE AND FUTURE PERSPECTIVE OF CANNABINOIDS IN GBM THERAPY}

The antineoplastic effects of cannabinoids have been investigated in a number of in vitro and in vivo studies (reviewed in Ladin et al., 2016). A pilot phase I clinical trial for the treatment of GBM patients indicated a good safety profile for THC (Velasco et al., 2007). The intra-tumor administration of THC in nine patients with actively growing recurrent GBM decreased tumor cell proliferation (Guzmán et al., 2006) and induced apoptosis (Carracedo et al., 2006). In contrast, cannabinoids promoted the survival of healthy oligodendrocytes (Molina-Holgado et al., 2002), astrocytes (Gómez Del Pulgar et al., 2002), and neurons (Howlett et al., 2002; Mechoulam, 2002). A tumor-specific cytostatic/cytotoxic effect of cannabinoids would, therefore, have great relevance for the treatment of GBM.

Pre-clinical studies have also investigated the anti-tumor effects of cannabinoid combinations (in particular THC:CBD) and found that the anti-neoplastic effect of THC was enhanced when combined with CBD (reviewed in Ladin et al., 2016). The therapeutic potential of THC:CBD combinations was, furthermore, tested in combination with standard GBM chemotherapy, such as the alkylating anti-neoplastic drug TMZ or with ionizing radiotherapy. In a GBM xenograft model in nude mice, the reduction of tumor size could be enhanced by co-administration of THC with CBD and TMZ in comparison to the effects of THC, CBD and TMZ alone (Torres et al., 2011). In a further study, THC:CBD co-treatment of orthotopic GBM tumors in C57BL/6 mice enhanced the killing effect of ionizing radiation (Scott et al., 2014; Ladin et al., 2016).

These beneficial effects of THC:CBD preparations in pre-clinical models have led to a placebo-controlled phase II clinical trial investigating a THC:CBD mixture in combination

\section{REFERENCES}

Adamson, C., Kanu, O. O., Mehta, A. I., Di, C., Lin, N., Mattox, A. K., et al. (2009). Glioblastoma multiforme: a review of where we have been and where we are going. Expert Opin. Investig. Drugs 18, 1061-1083. doi: 10.1517/13543780903052764

Aguado, T., Carracedo, A., Julien, B., Velasco, G., Milman, G., Mechoulam, R., et al. (2007). Cannabinoids induce glioma stem-like cell differentiation and inhibit gliomagenesis. J. Biol. Chem. 282, 6854-6862. doi: 10.1074/jbc. M608900200

Blázquez, C., Casanova, M. L., Planas, A., Gómez Del Pulgar, T., Villanueva, C., Fernández-Aceüero, M. J., et al. (2003). Inhibition of tumor angiogenesis by cannabinoids. FASEB J. 17, 529-531. doi: 10.1096/fj.02-0795fje

Blázquez, C., Carracedo, A., Salazar, M., Lorente, M., Egia, A., GonzálezFeria, L., et al. (2008a). Down-regulation of tissue inhibitor of metalloproteinases-1 in gliomas: a new marker of cannabinoid antitumoral with dose-intense TMZ in GBM patients (clinical trial NCT01812603). The company GW Pharmaceuticals reported in their orphan drug-designated study positive results in the treatment of GBM (Schultz and Beyer, 2017; Schultz, 2018). This study included 21 adult patients with histopathologicallyconfirmed GBM and with a Karnofsky performance scale of $60 \%$ or greater (clinical trial NCT01812603; Schultz and Beyer, 2017). Patients received orally a maximum of 12 sprays per day delivering $100 \mu \mathrm{l}$ of a solution containing $27 \mathrm{mg} / \mathrm{ml} \mathrm{THC}$ and $25 \mathrm{mg} / \mathrm{ml} \mathrm{CBD}$. The control group received TMZ only and had a $44 \%$ 1-year survival rate. In contrast the THC:CBD plus TMZ group showed a $83 \%$ 1-year survival rate with a median survival over 662 days compared with 369 days in the control group. (Schultz and Beyer, 2017; Schultz, 2018). These first results of clinical investigations are promising and point to the importance of cannabinoid translational research leading to clinically relevant studies. In the future, endocannabinoiddegrading MAGL enzyme might also be an interesting target since it changes the fatty acid network of cancer cells modulating their pathogenicity (Nomura et al., 2010).

In conclusion, cannabinoids show promising anti-neoplastic functions in GBM by targeting multiple cancer hallmarks such as resistance to programmed cell death, neoangiogenesis, tissue invasion or stem cell-induced replicative immortality. The effects of cannabinoids can be potentially enhanced by combination of different cannabinoids with each other or with chemotherapeutic agents. This requires, however, a detailed understanding of cannabinoid-induced molecular mechanisms and pharmacological effects. Ultimately, these findings might foster the development of improved therapeutic strategies against GBM and, perhaps, other diseases of the nervous system as well.

\section{AUTHOR CONTRIBUTIONS}

\section{CD, IES and MK wrote the manuscript.}

\section{ACKNOWLEDGMENTS}

This study was supported by the Werner Otto Stiftung (grant to $\mathrm{CD}$ and $\mathrm{MK}$; project 8/92).

activity? Neuropharmacology 54, 235-243. doi: 10.1016/j.neuropharm.2007 06.021

Blázquez, C., Salazar, M., Carracedo, A., Lorente, M., Egia, A., GonzálezFeria, L., et al. (2008b). Cannabinoids inhibit glioma cell invasion by down-regulating matrix metalloproteinase-2 expression. Cancer Res. 68, 1945-1952. doi: 10.1158/0008-5472.CAN-07-5176

Blázquez, C., González-Feria, L., Alvarez, L., Haro, A., Casanova, M. L., and Guzmán, M. (2004). Cannabinoids inhibit the vascular endothelial growth factor pathway in gliomas. Cancer Res. 64, 5617-5623. doi: 10.1158/0008-5472. can-03-3927

Brown, K. J., Laun, A. S., and Song, Z. H. (2017). Cannabidiol, a novel inverse agonist for GPR12. Biochem. Biophys. Res. Commun. 493, 451-454. doi: 10.1016/j.bbrc.2017.09.001

Carracedo, A., Lorente, M., Egia, A., Blázquez, C., García, S., Giroux, V., et al. (2006). The stress-regulated protein p8 mediates cannabinoid-induced apoptosis of tumor cells. Cancer Cell 9, 301-312. doi: 10.1016/j.ccr.2006.03.005 
Ciaglia, E., Torelli, G., Pisanti, S., Picardi, P., D’Alessandro, A., Laezza, C., et al. (2015). Cannabinoid receptor CB1 regulates STAT3 activity and its expression dictates the responsiveness to SR141716 treatment in human glioma patients' cells. Oncotarget 6, 15464-15481. doi: 10.18632/oncotarget.3895

De Jesús, M. L., Hostalot, C., Garibi, J. M., Sallés, J., Meana, J. J., and Callado, L. F. (2010). Opposite changes in cannabinoid CB1 and CB2 receptor expression in human gliomas. Neurochem. Int. 56, 829-833. doi: 10.1016/j.neuint. 2010.03.007

Ellert-Miklaszewska, A., Ciechomska, I., and Kaminska, B. (2013). Cannabinoid signaling in glioma cells. Adv. Exp. Med. Biol. 986, 209-220. doi: 10.1007/97894-007-4719-7_11

Espejo-Porras, F., Fernández-Ruiz, J., Pertwee, R. G., Mechoulam, R., and García, C. (2013). Motor effects of the non-psychotropic phytocannabinoid cannabidiol that are mediated by $5-\mathrm{HT}_{1 \mathrm{~A}}$ receptors. Neuropharmacology 75 , 155-163. doi: 10.1016/j.neuropharm.2013.07.024

Galanti, G., Fisher, T., Kventsel, I., Shoham, J., Gallily, R., Mechoulam, R., et al. (2008). Delta 9-tetrahydrocannabinol inhibits cell cycle progression by downregulation of E2F1 in human glioblastoma multiforme cells. Acta Oncol. 47, 1062-1070. doi: 10.1080/02841860701678787

Gertsch, J., Leonti, M., Raduner, S., Racz, I., Chen, J. Z., Xie, X. Q., et al. (2008). Beta-caryophyllene is a dietary cannabinoid. Proc. Natl. Acad. Sci. U S A 105, 9099-9104. doi: 10.1073/pnas.0803601105

Gómez Del Pulgar, T., De Ceballos, M. L., Guzmán, M., and Velasco, G. (2002). Cannabinoids protect astrocytes from ceramide-induced apoptosis through the phosphatidylinositol 3-kinase/protein kinase B pathway. J. Biol. Chem. 277, 36527-36533. doi: 10.1074/jbc.M205797200

Guzmán, M., Duarte, M. J., Blázquez, C., Ravina, J., Rosa, M. C., Galve-Roperh, I., et al. (2006). A pilot clinical study of $\Delta^{9}$-tetrahydrocannabinol in patients with recurrent glioblastoma multiforme. Br. J. Cancer 95, 197-203. doi: 10.1038/sj. bjc. 6603236

Hassan, S., Eldeeb, K., Millns, P. J., Bennett, A. J., Alexander, S. P., and Kendall, D. A. (2014). Cannabidiol enhances microglial phagocytosis via transient receptor potential (TRP) channel activation. Br. J. Pharmacol. 171, 2426-2439. doi: 10.1111/bph.12615

Hernández-Tiedra, S., Fabriàs, G., Dávila, D., Salanueva, I. J., Casas, J., Montes, L. R., et al. (2016). Dihydroceramide accumulation mediates cytotoxic autophagy of cancer cells via autolysosome destabilization. Autophagy 12, 2213-2229. doi: 10.1080/15548627.2016.1213927

Howlett, A. C., Barth, F., Bonner, T. I., Cabral, G., Casellas, P., Devane, W. A., et al. (2002). International Union of Pharmacology. XXVII. Classification of cannabinoid receptors. Pharmacol. Rev. 54, 161-202. doi: 10.1124/pr. 54.2.161

Kaplan, J. S., Stella, N., Catterall, W. A., and Westenbroek, R. E. (2017). Cannabidiol attenuates seizures and social deficits in a mouse model of Dravet syndrome. Proc. Natl. Acad. Sci. U S A 114, 11229-11234. doi: 10.1073/pnas. 1711351114

Ladin, D. A., Soliman, E., Griffin, L., and Van Dross, R. (2016). Preclinical and clinical assessment of cannabinoids as anti-cancer agents. Front. Pharmacol. 7:361. doi: 10.3389/fphar.2016.00361

Laun, A. S., and Song, Z. H. (2017). GPR3 and GPR6, novel molecular targets for cannabidiol. Biochem. Biophys. Res. Commun. 490, 17-21. doi: 10.1016/j.bbrc. 2017.05.165

Liebelt, B. D., Shingu, T., Zhou, X., Ren, J., Shin, S. A., and Hu, J. (2016). Glioma stem cells: signaling, microenvironment and therapy. Stem Cells Int. 2016:7849890. doi: 10.1155/2016/7849890

Louis, D. N., Perry, A., Reifenberger, G., von Deimling, A., Figarella-Branger, D., Cavenee, W. K., et al. (2016). The 2016 world health organization classification of tumors of the central nervous system: a summary. Acta Neuropathol. 131, 803-820. doi: 10.1007/s00401-016-1545-1

Lu, H. C., and Mackie, K. (2016). An introduction to the endogenous cannabinoid system. Biol. Psychiatry 79, 516-525. doi: 10.1016/j.biopsych.2015.07.028

Manini, I., Caponnetto, F., Bartolini, A., Ius, T., Mariuzzi, L., Di Loreto, C., et al. (2018). Role of microenvironment in glioma invasion: what we learned from in vitro models. Int. J. Mol. Sci. 19:E147. doi: 10.3390/ijms19010147

Marcu, J. P., Christian, R. T., Lau, D., Zielinski, A. J., Horowitz, M. P., Lee, J., et al. (2010). Cannabidiol enhances the inhibitory effects of delta9tetrahydrocannabinol on human glioblastoma cell proliferation and survival. Mol. Cancer Ther. 9, 180-189. doi: 10.1158/1535-7163.MCT-09-0407
Massi, P., Valenti, M., Solinas, M., and Parolaro, D. (2010). Molecular mechanisms involved in the antitumor activity of cannabinoids on gliomas: role for oxidative stress. Cancers 2, 1013-1026. doi: 10.3390/cancers2021013

Mechoulam, R. (1970). Marihuana chemistry. Science 168, 1159-1166. doi: $10.1126 /$ science.168.3936.1159

Mechoulam, R. (2002). Discovery of endocannabinoids and some random thoughts on their possible roles in neuroprotection and aggression. Prostaglandins Leukot. Essent. Fatty Acids 66, 93-99. doi: 10.1054/plef.2001. 0340

Molina-Holgado, E., Vela, J. M., Arévalo-Martin, A., Almazán, G., Molina-Holgado, F., Borrell, J., et al. (2002). Cannabinoids promote oligodendrocyte progenitor survival: involvement of cannabinoid receptors and phosphatidylinositol-3 kinase/Akt signaling. J. Neurosci. 22, 9742-9753. doi: 10.1523/JNEUROSCI.22-22-09742.2002

Nabissi, M., Morelli, M. B., Amantini, C., Liberati, S., Santoni, M., Ricci-Vitiani, L., et al. (2015). Cannabidiol stimulates Aml-1a-dependent glial differentiation and inhibits glioma stem-like cells proliferation by inducing autophagy in a TRPV2-dependent manner. Int. J. Cancer 137, 1855-1869. doi: 10.1002/ijc. 29573

Nabissi, M., Morelli, M. B., Santoni, M., and Santoni, G. (2013). Triggering of the TRPV2 channel by cannabidiol sensitizes glioblastoma cells to cytotoxic chemotherapeutic agents. Carcinogenesis 34, 48-57. doi: 10.1093/carcin/ bgs 328

Nomura, D. K., Long, J. Z., Niessen, S., Hoover, H. S., Ng, S. W., and Cravatt, B. F. (2010). Monoacylglycerol lipase regulates a fatty acid network that promotes cancer pathogenesis. Cell 140, 49-61. doi: 10.1016/j.cell.2009.11.027

Ostrom, Q. T., Bauchet, L., Davis, F. G., Deltour, I., Fisher, J. L., Langer, C. E., et al. (2014). The epidemiology of glioma in adults: a "state of the science" review. Neuro Oncol. 16, 896-913. doi: 10.1093/neuonc/nou087

Pertwee, R. G. (2005). "Pharmacological actions of cannabinoids," in Cannabinoids. Handbook of Experimental Pharmacology, ed. R. G. Pertwee (Berlin, Heidelberg: Springer), 1-51.

Petersen, G., Moesgaard, B., Schmid, P. C., Schmid, H. H., Broholm, H., Kosteljanetz, M., et al. (2005). Endocannabinoid metabolism in human glioblastomas and meningiomas compared to human non-tumour brain tissue. J. Neurochem. 93, 299-309. doi: 10.1111/j.1471-4159.2005.03013.x

Rocha, F. C., Dos Santos Júnior, J. G., Stefano, S. C., and Da Silveira, D. X. (2014). Systematic review of the literature on clinical and experimental trials on the antitumor effects of cannabinoids in gliomas. J. Neurooncol. 116, 11-24. doi: 10.1007/s11060-013-1277-1

Russo, E. B. (2017). Cannabidiol claims and misconceptions. Trends Pharmacol. Sci. 38, 198-201. doi: 10.1016/j.tips.2016.12.004

Salazar, M., Carracedo, A., Salanueva, I. J., Hernández-Tiedra, S., Lorente, M., Egia, A., et al. (2009). Cannabinoid action induces autophagy-mediated cell death through stimulation of ER stress in human glioma cells. J. Clin. Invest. 119, 1359-1372. doi: 10.1172/jci37948

Schley, M., Ständer, S., Kerner, J., Vajkoczy, P., Schüpfer, G., Dusch, M., et al. (2009). Predominant CB2 receptor expression in endothelial cells of glioblastoma in humans. Brain Res. Bull. 79, 333-337. doi: 10.1016/j. brainresbull.2009.01.011

Schultz, S. (2018). GW pharmaceuticals plc investor presentation-february 2018. Available online at: http://ir.gwpharm.com/static-files/e7afbad8-ab2c4c8a-8e21-b9d3a7d36c70

Schultz, S., and Beyer, M. (2017). GW pharmaceuticals achieves positive results in phase 2 proof of concept study in glioma. Available online at: http://ir.gwpharm.com/static-files/cde942fe-555c-4b2f-9cc9-f34d24c7ad27

Scott, K. A., Dalgleish, A. G., and Liu, W. M. (2014). The combination of cannabidiol and Delta9-tetrahydrocannabinol enhances the anticancer effects of radiation in an orthotopic murine glioma model. Mol. Cancer Ther. 13, 2955-2967. doi: 10.1158/1535-7163.mct-14-0402

Scott, K. A., Dennis, J. L., Dalgleish, A. G., and Liu, W. M. (2015). Inhibiting heat shock proteins can potentiate the cytotoxic effect of cannabidiol in human glioma cells. Anticancer Res. 35, 5827-5837.

Sharma, C., Al Kaabi, J. M., Nurulain, S. M., Goyal, S. N., Kamal, M. A., and Ojha, S. (2016). Polypharmacological properties and therapeutic potential of beta-caryophyllene: a dietary phytocannabinoid of pharmaceutical promise. Curr. Pharm. Des. 22, 3237-3264. doi: 10.2174/1381612822666160311 115226 
Singer, E., Judkins, J., Salomonis, N., Matlaf, L., Soteropoulos, P., Mcallister, S., et al. (2015). Reactive oxygen species-mediated therapeutic response and resistance in glioblastoma. Cell Death Dis. 6:e1601. doi: 10.1038/cddis.2014.566

Solinas, M., Massi, P., Cantelmo, A. R., Cattaneo, M. G., Cammarota, R., Bartolini, D., et al. (2012). Cannabidiol inhibits angiogenesis by multiple mechanisms. Br. J. Pharmacol. 167, 1218-1231. doi: 10.1111/j.1476-5381.2012. 02050.x

Solinas, M., Massi, P., Cinquina, V., Valenti, M., Bolognini, D., Gariboldi, M., et al. (2013). Cannabidiol, a non-psychoactive cannabinoid compound, inhibits proliferation and invasion in U87-MG and T98G glioma cells through a multitarget effect. PLoS One 8:e76918. doi: 10.1371/journal.pone.0076918

Soroceanu, L., Murase, R., Limbad, C., Singer, E., Allison, J., Adrados, I., et al. (2013). Id-1 is a key transcriptional regulator of glioblastoma aggressiveness and a novel therapeutic target. Cancer Res. 73, 1559-1569. doi: 10.1158/00085472.can-12-1943

Stupp, R., Mason, W. P., Van Den Bent, M. J., Weller, M., Fisher, B., Taphoorn, M. J., et al. (2005). Radiotherapy plus concomitant and adjuvant temozolomide for glioblastoma. N Engl. J. Med. 352, 987-996. doi: 10.1056/NEJMoa043330

Torres, S., Lorente, M., Rodríguez-Fornés, F., Hernández-Tiedra, S., Salazar, M., Garcia-Taboada, E., et al. (2011). A combined preclinical therapy of cannabinoids and temozolomide against glioma. Mol. Cancer Ther. 10, 90-103. doi: 10.1158/1535-7163.MCT-10-0688

Velasco, G., Carracedo, A., Blázquez, C., Lorente, M., Aguado, T., Haro, A., et al. (2007). Cannabinoids and gliomas. Mol. Neurobiol. 36, 60-67. doi: 10.1007/s12035-007-0002-5

Wu, X., Han, L., Zhang, X., Li, L., Jiang, C., Qiu, Y., et al. (2012). Alteration of endocannabinoid system in human gliomas. J. Neurochem. 120, 842-849. doi: 10.1111/j.1471-4159.2011.07625.x

Conflict of Interest Statement: The authors declare that the research was conducted in the absence of any commercial or financial relationships that could be construed as a potential conflict of interest.

Copyright (C) 2018 Dumitru, Sandalcioglu and Karsak. This is an open-access article distributed under the terms of the Creative Commons Attribution License (CC BY). The use, distribution or reproduction in other forums is permitted, provided the original author(s) and the copyright owner are credited and that the original publication in this journal is cited, in accordance with accepted academic practice. No use, distribution or reproduction is permitted which does not comply with these terms. 